\title{
Recherche expérimentale en Chirurgie buccale : principes, méthodes et résultats
}

\author{
Dard M \\ Institut Straumann, Bâle, Suisse \\ michel.dard@straumann.com
}

\section{Introduction}

La recherche en chirurgie expérimentale fait partie intégrante de la recherche qui examine et évalue les idées conventionnelles et innovantes en rapport avec la chirurgie. La recherche en chirurgie expérimentale est dédiée à la chirurgie animale et constitue l'étape obligatoire avant la recherche en chirurgie humaine qui se déroule le plus souvent dans le cadre d'études de recherche clinique. Les aspects fondamentaux du développement, du traitement et de la prévention des maladies sont communément étudiés sur des modèles animaux. Dans un concept de recherche médicale contemporaine, appliqué à la régénération osseuse, la recherche en chirurgie expérimentale correspond à la partie in vivo de la recherche dite préclinique qui inclut aussi la biologie moléculaire et cellulaire (in vitro) et la biomécanique (pratiquée dans ce cas ex vivo).

\section{Méthodes}

La recherche chirurgicale expérimentale comporte trois piliers :

- légal et réglementaire

- moral et éthique

- scientifique.

Les lois, règlements et normes qui régissent la recherche en chirurgie expérimentale seront évoquées.

La règle des 3Rs qui vise à donner un cadre moral, sera présentée et son application au sein d'un véritable code de conduite proposée. L'importance des comités d'éthique animaliers institutionnels sera soulignée.

La démarche scientifique fondée sur des règles généralement connues, mais très partiellement appliquées, sera détaillée selon le plan suivant:

- revue de la littérature internationale comme étape précédant la rédaction du protocole expérimental

- élaboration de l'hypothèse de travail

- définition des objectifs

- $\quad$ intérêt et valeur des variables soumises à l'étude

- choix du modèle animal dans l'une des catégories suivantes (induite, spontanée, négative, orpheline) en prenant, de plus, en compte le fait que les petits et grands modèles animaux sont complémentaires

- gestion des phases pré-, per- et post-opératoires et accompagnement vers l'issue

- obligation et adéquation de l'étude statistique (analyse de puissance, analyse descriptive et de variance)

- description des matériaux et dispositifs médicaux ainsi que des méthodes chirurgicales utilisées pour leur implantation en chirurgie osseuse

- décision sur les méthodes d'observation et d'analyse en partant du diagnostic vétérinaire clinique jusqu’à la génomique en passant par l’imagerie et la microscopie

- collecte, compilation, traitement et archivage des données brutes et des rapports

- publications dans les revues internationales à comité de lecture

\section{Résultats}

Le propos ci-dessus sera largement illustré par des exemples concrets tirés de l'expérience propre de l'auteur dans le domaine de la chirurgie animale buccale et maxillaire de régénération osseuse.

Les limites et opportunités offertes par la présente méthodologie en recherche chirurgicale expérimentale seront analysées et les avantages d’un programme expérimental complet comparés aux désavantages d'une étude unique et ponctuelle. 